\title{
Qualidade física e fisiológica de sementes de pimenta dedo de moça sob diferentes doses de adubo orgânico e mineral
}

\author{
Physical and physiological quality of girl finger pepper seeds under organic and mineral \\ fertilization \\ Calidad física y fisiológica de pimiento dedo de niña bajo diferentes dosis de fertilizante orgánico y \\ mineral
}

Recebido: 12/08/2021 | Revisado: 22/08/2021 | Aceito: 04/09/2021 | Publicado: 06/09/2021

Anderson Fabrício Messias Rocha
ORCID: https://orcid.org/0000-0002-7975-1071
Instituto Federal de Educação, Ciência e Tecnologia Goiano, Brasil
E-mail: andersonfabricioagronomia@ gmail.com
Wanderson Moreira dos Santos
ORCID: https://orcid.org/0000-0003-1036-1945
E-mail: agro.wandsantos@gmail.com
Filipe Beserra da Silva
Instituto Federal de Educação, Ciência e Tecnologia Goiano, Brasi
ORCID: https://orcid.org/0000-0002-3895-899X
E-mail: filipe.silva.agro@ gmail.com
Luís Sérgio Rodrigues Vale
Instituto Federal de Educação, Ciência e Tecnologia Goiano, Brasil
ORCID: https://orcid.org/0000-0002-6303-9063
E-mail: luis.sergio@ifgoiano.edu.br
Mônica Lau da Silva Marques
Instituto Federal de Educação, Ciência e Tecnologia Goiano, Brasil
ORCID: https://orcid.org/0000-0002-2794-0815
Renata de Castro Marques Carvalho
E-mail: monicallau@ifgoiano.edu.br
ORCID: https://orcid.org/0000-0003-1870-4885
Universidade Federal de Goiás, Brasil
E-mail: renatacastromarques@ @otmail.com

\section{Resumo}

O efeito de adubos orgânicos no que tange a qualidade de sementes ainda não é claro na literatura, sendo que os insumos orgânicos já fazem parte da realidade do campo e são usados por muitos agricultores. Neste sentido, objetivou-se com este estudo avaliar a qualidade física e fisiológica de sementes da pimenta dedo de moça sob diferentes doses de adubo orgânico e mineral em Ceres-GO. O experimento foi em blocos casualizados com a linhagem de pimenta IFET 1572 e seis tratamentos com doses de adubo: T0 - sem adubação; T1 - $15 \mathrm{tha}^{-1}$; T2 - $30 \mathrm{t}$ $\mathrm{ha}^{-1}$; T3 - $45 \mathrm{t} \mathrm{ha}^{-1}$ e T4 - $60 \mathrm{t} \mathrm{ha}^{-1}$ de esterco bovino e T5 - adubo mineral, sendo $334 \mathrm{~kg} \mathrm{ha}^{-1}$ de Ureia; $277 \mathrm{~kg} \mathrm{ha}^{-1} \mathrm{de}^{-}$ Termofosfato magnesiano e $144 \mathrm{~kg} \mathrm{ha}^{-1}$ de Cloreto de Potássio. As variáveis analisadas foram: grau de umidade; teste padrão de germinação; emergência; condutividade elétrica; peso de 1000 sementes e Índice de velocidade de emergência. Os dados foram submetidos à análise de variância e as médias comparadas pelo teste Tukey a $5 \%$ de probabilidade $(\mathrm{p} \leq 0,05)$. Para comparar os adubos orgânicos foi aplicado a análise de regressão. Houve diferenças significativas na qualidade física e fisiológica das sementes na emergência, índice de velocidade de emergência, teste padrão de germinação e peso de mil sementes sob diferentes doses de esterco bovino e mineral. Doses crescentes de esterco bovino interferiram negativamente na qualidade física e fisiológica das sementes a partir da aplicação de $15 \mathrm{t}$ $\mathrm{ha}^{-1}$. O uso de adubo bovino pode ser utilizado para produção de sementes de pimenta dedo de moça.

Palavras-chave: Capsicum Baccatum; Esterco bovino; Produção orgânica; Vigor de sementes.

\section{Abstract}

The effect of organic fertilizers regarding seed quality is still unclear in the literature, as organic insums are already part of the reality of the field and are used by many farmers. In this sense, the objective of this study was to evaluate the physical and physiological quality of girl finger pepper seeds under different doses of organic and mineral fertilizer in Ceres-GO. The experiment was carried out in randomized blocks with the pepper lineage IFET 1572 and six treatments with fertilizer doses: $\mathrm{T} 0$ - no fertilizer; $\mathrm{T} 1-15 \mathrm{tha}^{-1}$ of cattle manure; $\mathrm{T} 2-30 \mathrm{t} \mathrm{ha}^{-1}$ of cattle manure; 
$\mathrm{T} 3-45 \mathrm{t} \mathrm{ha}^{-1}$ of cattle manure; $\mathrm{T} 4-60 \mathrm{t} \mathrm{ha}^{-1}$ of cattle manure and T5 - mineral fertilizer mineral fertilizer, $334 \mathrm{~kg} \mathrm{ha}^{-1}$ of Urea; $277 \mathrm{~kg} \mathrm{ha}^{-1}$ of Magnesium Thermophosphate and $144 \mathrm{~kg} \mathrm{ha}^{-1}$ of Potassium Chloride. The variables analyzed were: degree of humidity; germination pattern test (TPG); emergency; Electric conductivity; 1000 seed weight and emergence speed index (IVE). Data were subjected to analysis of variance and means compared by Tukey test at $5 \%$ probability $(\mathrm{p} \leq 0.05)$. To compare the organic fertilizers, regression was applied. There were significant differences in the physical and physiological quality of the seeds in the emergence, emergence speed index, germination pattern and weight of one thousand seeds under different doses of bovine and mineral manure. Increasing doses of bovine manure negatively interfered with the physical and physiological quality of the seeds from the application of $15 \mathrm{tha}{ }^{-1}$. The use of bovine fertilizer can be used for the production of girl finger pepper seeds.

Keywords: Capsicum baccatum; Cattle manure; Organic production; Seed vigor.

\section{Resumen}

El efecto de los fertilizantes orgánicos en la calidad de la semilla aún no está claro en la literatura, y los insums orgánicos ya son parte de la realidad del campo y son utilizados por muchos agricultores. En este sentido, el objetivo de este estudio fue evaluar la calidad física y fisiológica de las semillas de pimienta dedo de niña bajo diferentes dosis de fertilizante orgánico y mineral en Ceres-GO. El experimento se realizó en bloques al azar con el linaje de pimiento IFET 1572 y seis tratamientos con dosis de fertilizante: T0 - sin fertilizante; T1 - $15 \mathrm{t} \mathrm{ha}^{-1}$ de estiércol de ganado; T2 30 t ha ${ }^{-1}$ de estiércol de ganado; T3 - $45 \mathrm{t} \mathrm{ha}^{-1}$ de estiércol de ganado; T4 - $60 \mathrm{t} \mathrm{ha}^{-1}$ de estiércol de ganado y T5 fertilizante mineral fertilizante mineral, $334 \mathrm{~kg} \mathrm{ha}^{-1}$ de urea; $277 \mathrm{~kg} \mathrm{ha}^{-1}$ de Termofosfato de Magnesio y $144 \mathrm{~kg}^{-1}$ de Cloruro de Potasio. Las variables analizadas fueron: grado de humedad; prueba de patrón de germinación (TPG); emergencia; Conductividad eléctrica; Peso de 1000 semillas y índice de velocidad de emergencia (IVE). Los datos se sometieron a análisis de varianza y se compararon las medias mediante la prueba de Tukey al $5 \%$ de probabilidad ( $\mathrm{p} \leq$ 0,05). Para comparar los fertilizantes orgánicos se aplicó regresión. Hubo diferencias significativas en la calidad física y fisiológica de las semillas en la emergencia, índice de velocidad de emergencia, patrón de germinación y peso de mil semillas bajo diferentes dosis de estiércol bovino y mineral. El aumento de las dosis de estiércol bovino interfirió negativamente con la calidad física y fisiológica de las semillas a partir de la aplicación de $15 \mathrm{t}$ ha ${ }^{-1}$. El uso de fertilizante bovino se puede utilizar para la producción de semillas de pimienta dedo de niña.

Palabras clave: Capsicum baccatum; Estiércol bovino; Producción orgánica; Vigor de la semilla.

\section{Introdução}

As espécies de pimentas do gênero Capsicum pertencem à família Solanaceae. Dentre as espécies do gênero Capsicum, cinco são domesticadas e largamente cultivadas e utilizadas pelo homem: Capsicum annuum; C. bacccatum; C. chinense; $C$. frutescens e $C$. pubescens. A altura e forma de crescimento destas plantas variam de acordo com a espécie e as condições de cultivo. As espécies desse gênero são autógamas, ou seja, o pólen e o óvulo que é fecundado pertencem a uma mesma flor, podendo ocorrer também a polinização cruzada Costa et al. 2007).

O negócio de pimentas é um importante segmento do mercado agrícola brasileiro, com forte expressão na indústria alimentícia, farmacêutica e cosmética (Assunção, 2013). Apresenta características importantes para a alimentação humana, sendo os frutos ricos em vitamina C, compostos antioxidantes e bioativos (Borges, et al. 2020).

Praticamente em todas as regiões do país há cultivo de pimentas, no qual a agricultura familiar assume destaque na produção, uma vez que a cultura demanda muita mão de obra no seu processo produtivo e também, geralmente trabalhada em pequenas áreas de produção (Costa \& Henz 2007, Lopes, et al. 2016). A produção brasileira de pimentas quando comparadas a outras culturas é relativamente pequena. A produção nacional está em torno de 280.000 toneladas ao ano, com área plantada de 13.000 mil hectares (Rodrigues, 2016). Contudo, é uma cultura que gera empregos, renda e melhora a qualidade de vida dos agricultores familiares, assumindo importância econômica e social (Assunção, 2013).

Alves et al. (2005), destacam que o uso de insumos orgânicos é uma opção usada pelos agricultores para baratear o processo de produção de diversos cultivos que visam alcançar maior produção. Além disso, a prática de se produzir a própria semente para novos cultivos é também muito usada pelos agricultores, cujos processos de produção pode afetar a qualidade ou a produção de sementes (Pesagro-Rio, 2018).

Dessa forma, no que tange a produção e qualidade de sementes, o uso de adubo orgânico já é uma realidade no campo, no qual estudos vem demonstrando efeitos positivos, tanto no desenvolvimento da cultura quanto na melhora do 
rendimento de sementes (Carvalho, et al., 2017, Rech, Franke \& Barros, 2006, Santos et. al. 2017).

Todavia, a adubação deve seguir e atender as necessidades de cada espécie, uma vez que a reposta para cada material é diferente, podendo influenciar no desenvolvimento da cultura e na produção de suas sementes (Zucareli, et al. 2012). Visto sua composição nutricional, o efeito dos adubos orgânicos no solo já é bem conhecido, porém ainda pouco se conhece o efeito que seu uso pode influenciar na qualidade das sementes (Alves, et al. 2005).

Neste contexto, o objetivou-se avaliar a qualidade física e fisiológica de sementes de pimenta dedo de moça sob diferentes doses de adubo orgânico e mineral, em Ceres-GO.

\section{Metodologia}

O trabalho foi realizado no laboratório de Análise de Sementes do Instituto Federal Goiano - Campus Ceres. Foi utilizada sementes da linhagem de pimenta IFET 1572 "Dedo de moça" (Capsicum baccatum).

As sementes utilizadas nas análises físicas e fisiológicas tiveram origem de um experimento realizado na área experimental do IF Goiano - Campus Ceres, no período de janeiro a agosto de 2017. O delineamento experimental foi em blocos casualizados com seis tratamentos com doses de adubo: T0 - Controle (sem adubação); T1 - $15 \mathrm{t} \mathrm{ha}^{-1}$ de esterco bovino; T2 - $30 \mathrm{t} \mathrm{ha}^{-1}$ de esterco bovino; T3 - $45 \mathrm{t} \mathrm{ha}^{-1}$ de esterco bovino e T4 $-60 \mathrm{t} \mathrm{ha}^{-1}$ de esterco bovino e T5 com adubo mineral, sendo $334 \mathrm{~kg} \mathrm{ha}^{-1}$ de Ureia; $277 \mathrm{~kg} \mathrm{ha}^{-1}$ de Termofosfato magnesiano e $144 \mathrm{~kg} \mathrm{ha}^{-1}$ de Cloreto de Potássio contendo quatro repetições. Foi utilizada a linhagem de pimenta Dedo de Moça IFET 1572 oriunda do Programa de Melhoramento Genético do IF Goiano - Campus Ceres.

Os dados de precipitação pluviométrica e temperatura média durante a condução do experimento encontram-se na Figura 1. Esses dados foram obtidos próximo à área experimental, na estação meteorológica do Instituto Federal Goiano Campus Ceres, Ceres GO.

Figura 1. Precipitação e temperatura média mensal nos meses de janeiro a agosto de 2017 em Ceres, GO.

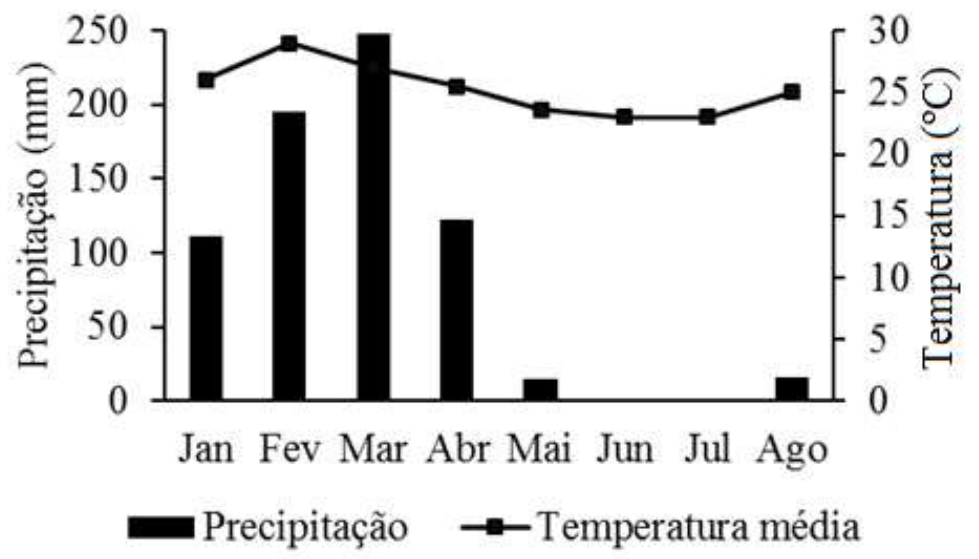

Fonte: Autores.

O solo da área experimental foi classificado como Latossolo vermelho (Embrapa, 2013), com as seguintes características químicas e físicas de acordo com a análise de solo, na profundidade de 0-20 cm: $438 \mathrm{~g} \mathrm{~kg}^{-1} \mathrm{de}^{\text {Argila; } 523 \mathrm{~g} \mathrm{~kg}^{-1}}$ de Areia e $39 \mathrm{~g} \mathrm{~kg}^{-1}$ de Silte; pH em água (1:25) 6,41; 69,45 $\mathrm{mg} \mathrm{dm}^{-3} \mathrm{de} \mathrm{P}$ (Mehlich); 145,5 mg dm${ }^{-3} \mathrm{de} \mathrm{K}^{+}$; 3,36 $\mathrm{Cmol}_{\mathrm{c}} \mathrm{dm}^{-3}$ de $\mathrm{Ca}^{2+} ; 1,1 \mathrm{Cmol}_{\mathrm{c}} \mathrm{dm}^{-3}$ de $\mathrm{Mg}^{2+} ; 1,5 \mathrm{Cmol}_{\mathrm{c}} \mathrm{dm}^{-3} \mathrm{de} \mathrm{H}+\mathrm{Al} ; 76,2 \%$ de saturação por bases (V\%) e 22,2 $\mathrm{g} \mathrm{dm}^{-3}$ de matéria orgânica. 
As mudas de pimentas foram produzidas em casa de vegetação com substrato comercial Plantmax ${ }^{\circledR}$, em embalagens descartáveis de $200 \mathrm{~mL}$. As mudas foram transplantadas de forma manual quando apresentaram de 6 a 12 folhas definitivas, cerca de 60 dias após a semeadura. A área foi preparada de forma convencional, com uma aração e duas gradagens.

O adubo orgânico foi aplicado na cova 15 dias antes do plantio e apresentava as seguintes características, com base na

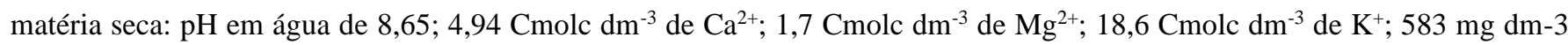
de P e $17 \mathrm{~g} \mathrm{~kg}^{-1}$ de N; 13,2\% de matéria orgânica. No tratamento com adubo mineral foi utilizado $334 \mathrm{~kg} \mathrm{ha}^{-1}$ de Ureia; $277 \mathrm{~kg}$ $\mathrm{ha}^{-1}$ de Termofosfato magnesiano e $144 \mathrm{~kg} \mathrm{ha}^{-1}$ de Cloreto de Potássio no plantio, conforme recomendações de Ribeiro et al. (2008). A adubação de cobertura foi parcelada em 6 aplicações, a cada 30 dias. Para a adubação de cobertura nos tratamentos com adubação orgânica foram aplicados $120 \mathrm{~kg} \mathrm{ha}^{-1} \mathrm{de} \mathrm{N}$, como fonte o esterco bovino (quantidade de esterco calculada com base no N disponível no esterco: 1,7\%).

A área de cada parcela era correspondente a de $20 \mathrm{~m}^{2}(5 \times 4 \mathrm{~m})$. O espaçamento foi de $1 \mathrm{~m} \mathrm{x} 1 \mathrm{~m}$, totalizando 20 plantas por parcela. Foram utilizadas as duas linhas centrais para análise de frutos. O ciclo fenológico da pimenta foi de 210 dias após o transplante (DAT). As colheitas foram realizadas aproximadamente a cada 15 dias, totalizando seis colheitas, com início aos 95 dias após o transplante.

Foram utilizados 20 frutos maduros de cada parcela, totalizando 80 frutos por tratamento. As sementes utilizadas para análise foram oriundas da quarta colheita de frutos. As sementes foram extraídas de forma manual, com auxílio de uma faca de cozinha e pré-secadas por três dias à sombra em temperatura ambiente e em estufa por $24 \mathrm{~h}$ a $48{ }^{\circ} \mathrm{C}$ e armazenadas sob refrigeração em sacos de papel a 4-6 ${ }^{\circ} \mathrm{C}$ (Brasil, 2009). No Laboratório de Análises de Sementes foram realizados os seguintes testes: Pureza física; Grau de Umidade; Teste Padrão de Germinação (TPG); Condutividade Elétrica; Peso de 1000 sementes e Índice de Velocidade de Emergência (IVE), através de análises metodológica quantitativa (Köche, 2011).

O Grau de Umidade (\%) foi determinado utilizando o método da estufa a $105 \pm 3{ }^{\circ} \mathrm{C}$, por 72 horas. Foram utilizadas oito repetições de 100 sementes por tratamento, e os resultados expressos em gramas (Brasil, 2009). Paralelamente foi determinado o peso de matéria seca $\left(100 \mathrm{mg}\right.$ sementes $\left.{ }^{-1}\right)$.

O Teste Padrão de Germinação (TPG) (\%) foi realizado com quatro subamostras de 50 sementes cada e distribuídas sobre duas folhas de papel mata borrão umedecidas com água deionizada com volume equivalente a 2,5 vezes o peso do papel seco, em caixas gerbox. As caixas foram mantidas em germinador com temperatura alternada $\left(20-30{ }^{\circ} \mathrm{C}\right)$ e com fotoperíodo de oito horas para a maior temperatura (Torres, 2005). As avaliações foram feitas no $14^{\circ}$ dia após da implantação (Brasil, 2009).

A Condutividade Elétrica (CE) de sementes foi determinada com quatro subamostras de 50 sementes para cada tratamento, as quais foram pesadas em balança analítica de precisão $(0,001 \mathrm{~g})$ e colocadas em copos descartáveis com $25 \mathrm{~mL}$ de água destilada, mantidos em câmara do tipo B.O.D. a $25{ }^{\circ} \mathrm{C}$ por 24 horas (Bhering et al. 2006). Após, a condutividade elétrica foi determinada em condutivímetro de bancada e os resultados expressos em $\mu \mathrm{Scm}^{-1} \mathrm{~g}^{-1}$ de sementes.

O Peso de 1000 sementes (g) foi realizado com oito subamostras de 100 sementes e pesadas em balança de precisão (Brasil, 2009).

A Emergência de plântulas (\%) foi feita em sementeiras com areia em casa de vegetação com sistema de microaspersão. Foram utilizadas quatro repetições de 50 sementes. Foi computada a porcentagem de plântulas normais aos 30 dias após o início do teste.

O Índice de Velocidade de Emergência (IVE) foi calculado de acordo com Maguire (1962), IVE= N1/D1 +N2/D2 + $\ldots .+\mathrm{Nn} / \mathrm{Dn}$, Onde: IVE = índice de velocidade de emergência; $\mathrm{N}=$ números de plântulas verificadas no dia da contagem; $\mathrm{D}=$ números de dias após a semeadura em que foi realizada a contagem. Os dados utilizados formam o teste de emergência de plântulas.

Os dados obtidos foram submetidos à análise de variância e quanto significativos feita a análise regressão para as 
médias das doses de adubo orgânico. Para comparar os resultados quanto a adubação mineral com adubo orgânico aplicou-se o teste de Tukey a $5 \%$ de probabilidade $(\mathrm{p} \leq 0,05)$, utilizando o programa estatístico SISVAR 5.6.

\section{Resultados e Discussão}

Os resultados do teste de Tukey $(\mathrm{p} \leq 0,05)$ diferiram significativamente para emergência de plântulas, índice de velocidade de emergência, peso de mil sementes e teste padrão de germinação (TPG). Não houve diferença significativa para condutividade elétrica, massa seca e grau de umidade de sementes (Tabela 1).

Teores de água muito baixos menores ou iguais a $10 \%$, ou muito altos, maior ou igual a $17 \%$, podem influenciar significativamente nos resultados das análises de condutividade elétrica, como é citado por Vieira et al. (2002). O grau de umidade observado no presente estudo encontra-se na faixa de 9\% (Tabela 1). Completa-se ainda que, a uniformidade no grau de umidade é imprescindível para padronização das avaliações dos testes de qualidade de sementes para obtenção de resultados consistentes (Marco Filho, 1999).

Tabela 1: Emergência (EMER), índice de velocidade de emergência (IVE), condutividade elétrica (COND), massa seca de sementes (MSEC), peso de mil sementes (PMIL), teste padrão de germinação de sementes (TPG) e grau de umidade de sementes (Umd) de pimenta dedo de moça submetidos a adubação orgânica e mineral. Ceres, GO. 2017.

\begin{tabular}{llllllll}
\hline Trat $\left(\mathrm{t} \mathrm{ha}^{-1}\right)$ & EMER & IVE & COND & MSEC & PMIL & TPG & Umd \\
\hline 0 & $46,5 \mathrm{ab}$ & $2,56 \mathrm{ab}$ & $3039,503 \mathrm{a}$ & $0,1511 \mathrm{a}$ & $3,12 \mathrm{~d}$ & $59,00 \mathrm{a}$ & $9,66 \mathrm{a}$ \\
15 & $63,5 \mathrm{a}$ & $3,62 \mathrm{a}$ & $3864,452 \mathrm{a}$ & $0,1781 \mathrm{a}$ & $4,00 \mathrm{a}$ & $50,75 \mathrm{ab}$ & $9,07 \mathrm{a}$ \\
30 & $56,5 \mathrm{ab}$ & $3,13 \mathrm{ab}$ & $2665,642 \mathrm{a}$ & $0,1601 \mathrm{a}$ & $3,62 \mathrm{bc}$ & $40,00 \mathrm{bc}$ & $10,24 \mathrm{a}$ \\
& & & & & & & \\
45 & $51,0 \mathrm{ab}$ & $2,76 \mathrm{ab}$ & $2410,88 \mathrm{a}$ & $0,1708 \mathrm{a}$ & $3,67 \mathrm{~b}$ & $40,75 \mathrm{bc}$ & $8,15 \mathrm{a}$ \\
60 & $36,5 \mathrm{~b}$ & $2,08 \mathrm{~b}$ & $2410,88 \mathrm{a}$ & $0,1595 \mathrm{a}$ & $3,39 \mathrm{c}$ & $35,75 \mathrm{~b}$ & $9,09 \mathrm{a}$ \\
Mineral & $53,5 \mathrm{ab}$ & $2,90 \mathrm{ab}$ & $3204,818 \mathrm{a}$ & $0,1600 \mathrm{a}$ & $3,42 \mathrm{bc}$ & $60,25 \mathrm{a}$ & $9,00 \mathrm{a}$ \\
\hline CV $(\%)$ & 18,05 & 17,15 & 26,54 & 8,01 & 8,5 & 17,72 & 14,97 \\
\hline
\end{tabular}

Nota. Médias seguidas pela mesma letra na coluna, não diferem entre si pelo teste de Tukey a $5 \%(\mathrm{p} \leq 0,05)$ de probabilidade. Fonte: Autores.

Para a variável EMER, IVE através do teste de Tukey $(\mathrm{p} \leq 0,05)$ observa-se que os resultados para a dose de $15 \mathrm{t} \mathrm{ha}^{-1}$ (63,5\% e 3,62, respectivamente) foram superiores à dose de $60 \mathrm{t} \mathrm{ha}^{-1}$ (36,5\% e 2,08, respectivamente) e igual às demais doses de esterco e ao adubo mineral. As demais doses não diferiram entre si. Nota-se que mesmo apresentando resultados estatisticamente diferentes entre os tratamentos, o percentual de germinação ficou abaixo de $60 \%$. Esses resultados podem ser explicados pelas características naturais da semente, como pontua Marques et al. (2020), Venturin et al. (2014) e Finch-Savage \& Leubner-Metzger (2006), que sementes de pimentas do gênero Capsicum apresentam naturalmente dormência mesmo sob condições favoráveis, o que pode comprometer a germinação e emergências das plântulas. A dormência é considerada um mecanismo de adaptação das sementes contra modificações no ambiente que impossibilitam ou dificultam as reações metabólicas naturais durante o processo de desenvolvimento da semente, sendo que a retomada do crescimento do embrião só é restabelecida após a ação de um estímulo ambiental específico Marcos Filho (2005).

O peso de mil sementes (PMIL) foi maior na dose de $15 \mathrm{t} \mathrm{ha}^{-1}$, quando comparado aos demais tratamentos (Tabela 1). Resultado diferente foi obtido por Rech, Franke \& Barros (2006), em que doses menores de adubo orgânico tiveram baixo 
rendimento no peso de mil sementes para a cultura da abobrinha, no entanto, o uso de dose duas vezes maior ao recomendado para a cultura, apresentou decréscimo para esta variável.

A análise de regressão para as doses crescentes de esterco bovino não apresentou diferenças significativas para a qualidade fisiológica de sementes quanto a condutividade elétrica e grau de umidade. Para as demais variáveis, teste padrão de germinação (TPG), emergência (EMER), peso de mil sementes (PMIL) índice de velocidade de emergência (IVE), houve significância na análise de regressão das doses crescentes de esterco bovino.

O comportamento do TPG foi decrescente, ajustando-se a uma função linear, cujo efeito dos tratamentos pode ser explicado na ordem de 89,32\% pelos tratamentos utilizados. Assim, de acordo com a derivada da função de TPG obtida, o maior resultado para esta variável $(56,55)$ é atingido sem a aplicação de adubos nesta cultura. Conforme houve a aplicação de esterco bovino, houve então a diminuição do TPG (Figura 2).

Figura 2. Teste padrão de germinação de sementes de Capsicum baccatum sob doses de esterco bovino.

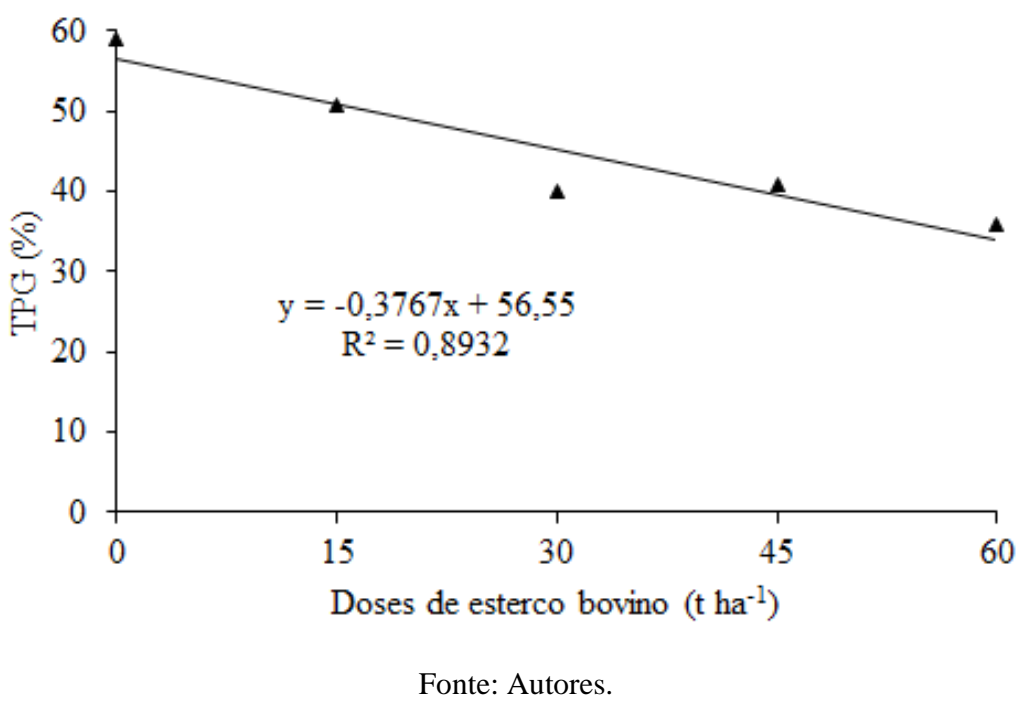

Esse resultado pode ser explicado pelo aumento da concentração de nitrogênio na adubação que pode ter influenciado negativamente na qualidade das sementes (Imolesi, et al., 2001), o que pode justificar o decréscimo na emergência conforme aumenta-se a dose de esterco bovino.

Nota-se na Figura 3A, 3B e 3C para as variáveis: peso de mil sementes, emergência de plântulas e índice de velocidade de emergência que os dados se ajustaram ao modelo quadrático. Houve decréscimo na qualidade das sementes de pimenta a partir da primeira dose de esterco bovino. 
Figura 3. Emergência de plântulas (A), peso de 1000 sementes (B) e índice de velocidade de emergência (C) de plântulas de Capsicum baccatum, sob doses de esterco bovino.
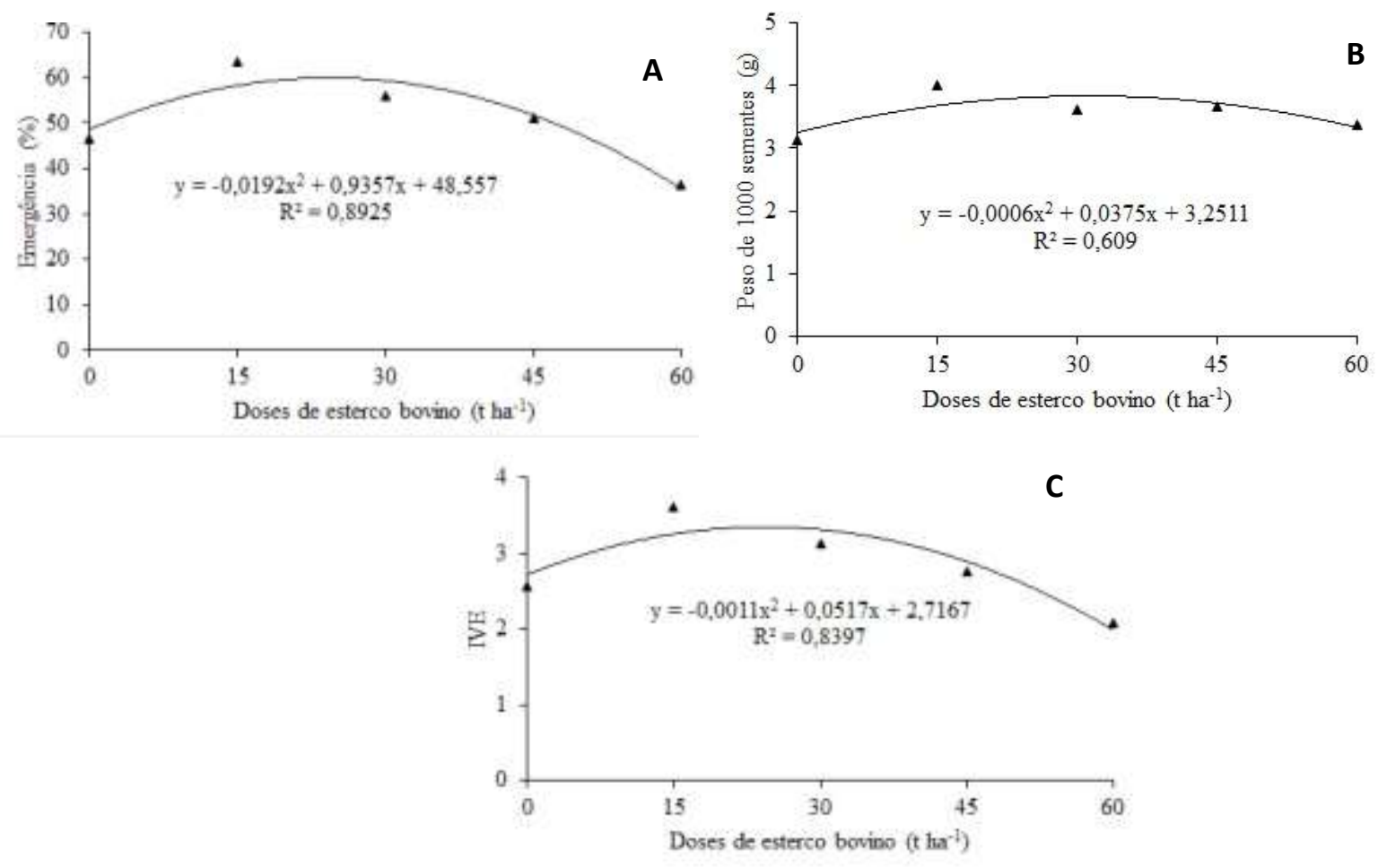

Fonte: Autores.

De acordo com a derivada da função para emergência de plântulas (Figura 3A) o maior percentual de germinação $(59,95 \%)$ é atingido na dose de $24,37 \mathrm{tha}^{-1}$ de esterco bovino.

O maior peso de mil sementes (4 g) (Figura 3B) seria atingindo aplicando-se a dose de 32,50 $\mathrm{t} \mathrm{ha}^{-1}$ de esterco bovino, e o melhor IVE (2,71) (Figura 3C) seria atingido com aplicação de 23,5 $\mathrm{t} \mathrm{ha}^{-1}$ de esterco bovino. Dessa forma, para as respectivas variáveis, o padrão de comportamento, sob doses de esterco bovino teve uma melhora na qualidade com a aplicação de esterco.

Neste sentido, a redução da qualidade de sementes pelo aumento das doses de esterco bovino provavelmente pode ter ocorrido devido a aumento nas concentrações de nitrogênio, que pode afetar a qualidade das sementes, visto que altera o teor de proteínas das sementes e promovem redução no seu vigor (Imolesi, et al. 2001, Warraich, et al. 2002). Além disso, altas concentrações de nitrogênio interferem na absorção do fósforo afetando a disponibilidade de energia pela planta em função da competição entre o fósforo e o nitrogênio pelo mesmo sítio de absorção (Marmaril \& Miller, 1970).

\section{Conclusão}

O grau de umidade e a condutividade elétrica não foram influenciados pelas doses de esterco bovino e adubo mineral. A dose de adubo bovino que proporcionou melhor qualidade fisiológica das sementes de pimenta dedo de moça em relação à maioria dos parâmetros estudados foi a de $15 \mathrm{tha}^{-1}$.

Doses elevadas de esterco bovino afeta negativamente a qualidade fisiológica de sementes de pimenta dedo de moça.

$\mathrm{O}$ uso de adubo bovino pode ser utilizado para produção de sementes de pimenta dedo de moça. Contudo, se faz necessário novos estudos para uma investigação mais aprofundada sobre o efeito do esterco nas características químicas decorrido de doses crescentes de esterco bovino na produção de sementes de pimenta dedo de moça. 


\section{Referências}

Alves, E. U., Oliveira, A. P., Bruno, R. L. A., Sader, R. \& Alves, A. U. (2005). Rendimento e qualidade fisiológica de sementes de coentro cultivado com adubação orgânica e mineral. Revista Brasileira de Sementes, 27(1), 132-137. https://doi.org/10.1590/S0101-31222005000100016

Assunção, P. V. (2013). Dispêndios e viabilidade econômica da produção de pimenta no sul de Goiás. Revista Política Agrícola, $22,110-118$.

Bhering, M. C., Dias, D. C. F. S. \& Vidigal, D. S. P. N. (2006). Teste de envelhecimento acelerado em sementes de pimenta. Revista Brasileira de Sementes, 28(3), 64-71. https://doi.org/10.1590/S0101-31222006000300010

Borges, R. B. G., Morais, R. A., Soares, C. M. S., Santos, A. L., Martins, G. A. S., \& Silva, J. F. M. da. (2020). Bioactive compounds of pineapple (Pineapple comosus) and girl finger pepper (Capsicum baccatum) and their correlation with antioxidant activity. Research, Society and Development, 9(7), e71973210. https://doi.org/10.33448/rsd-v9i7.3210

Brasil. Ministério da Agricultura, Pecuária e Abastecimento. (2009). Regras para análise de sementes / Ministério da Agricultura, Pecuária e Abastecimento. Secretaria de Defesa Agropecuária. Mapa/ACS. 399 p.

Carvalho, J. B., Mota, J. M. N., Alvarenga, C. B., Maciel, G. M., Silva, A. A. \& Borba, M. E. A. (2017). Produção e qualidade fisiológica de sementes de alface cultivada com adubação orgânica e mineral. Revista de Ciências Agrárias - Amazonian Journal of Agricultural and Environmental Scie20nces. 60(1), 70 - 76. https://doi.org/10.4322/rca. 2424

Costa, C. S. R., \& Henz, G. P. (2007). Pimenta (Capsicum spp.). Embrapa Hortaliças. Sistemas de Produção, 2. http://sistemasdeproducao.cnptia.embrap a.br/FontesHTML/Pimenta/Pimenta_capsicum_spp/cultivares.html

Finch-Savage W. E., \& Leubnermetzger G. (2006) Seed dormancy and the control of germination. New Phytologist, Lancaster, $171,501-523$.

Imolesi, A. S., Pinho, E. V. R. V.; Vieira, M. D. G. G. C. \& Corrêa, R. S. B. Influência da adubação nitrogenada na qualidade fisiológica das sementes te milho. Ciência e Agrotecnologia, 25(5), 1119-11126, 2001. https://www.researchgate.net/profile/Renzo-Pinho/publication/237275812_influencia_da_adu bacao_nitrogenada_na_qualidade_fisiologica_das_sementes_de_milho/links/02e7e538e0992be269000000/influencia-da-adubacao-nitrogenada-na-qualidadefisiologica-das-sementes-de-milho.pdf

Köche, J. C. (2011). Fundamentos de metodologia científica: teoria da ciência e iniciação à pesquisa. Editora Vozes.

Lopes, M. A. P., Gonçalves, L. D. \& Morais, E. G. (2016). Caracterização de acessos de pimenta cumari de distribuição natural para fins de melhoramento genético. Revista Agrogeoambiental. 8(4), 105-115. https://doi.org/10.18406/2316-1817v8n42016898

Maguire, J. D. (1962). Speed germination-aid in selection and evaluation for seedling emergence and 102 vigor. Crop Sciense, $2,176-177$.

Marcos Filho, J. Testes de vigor: importância e utilização. In: Krzyzanowski, F. C., Vieira, R. D. \& França Neto, J. B. (Eds.). (1999). Vigor de sementes: conceitos e testes. ABRATES, p. 1- 24.

Marmaril, C. P. \& Miller, M. H. (1970). Effects of ammonium on the uptake of phosphorus, sulfur, and rubidium by corn. Agronomy Journal, 62(6), 753-758.

Marques, M. L. S., Vale, L. S. R., Dias, L. J., Moreno, V. S., Pedrosa, J. A., Jesus, J. M. I., Santos, W. M., Cruz, D. R. C., Martins, A. L. S., \& Marques, V. S. (2020). Breaking dormance of 'Cumari Verdadeira' pepper seeds. Research, Society and Development, 9(9), e957998149. https://doi.org/10.33448/rsdv9i9.8149.

Pesagro-Rio. (2018). Empresa de Pesquisa Agropecuária do Estado do Rio de Janeiro. Catálogo de Sementes produzidas sob manejo orgânico. 1, Pesagro-Rio e UFRRJ. http://www.ecoagri.com.br/web/wp-content/uploads/Web-Catalogo-de-sementes-organicas.pdf

Rech, E. G., Franke, L. B \& Barros, (2006). Adubação orgânica e mineral na produção de sementes de abobrinha. Rev. bras. sementes, 28(2), 110-116. https://doi.org/10.1590/S0101-31222006000200014

Ribeiro, C. S. C., Lopes, C. A., Carvalho, S. I. C., Henz, G. P. \& Reifschneider, F. J. B. (2008). Pimentas Capsicum. Embrapa Hortaliças, (21a ed.), 200 p.

Rodrigues, P. Pimenta (2016). A Lavoura, n. 716, https://www.embrapa.br/documents/1355126/10765216/2016_11_01+Revista+A+Lavoura++Pimenta+\%28NCO+via+AE\%29.pdf/cb54ee6c-6de0-4a4d-ab8c-1137a38a05f9.

Santos, L. M., lima, V. L. A., Azevedo, C. A. V., Lima, R. L. S., Silva, A. A. R. \& Nóbrega, R. A. (2017). Crescimento e número de sementes de pinhãomanso submetido a adubação orgânica e mineral. Revista brasileira de agricultura irrigada, 11(1), 1233-1243. https://doi.org/10.7127/RBAI.V11N100572

Torres, S. B. (2005). Envelhecimento acelerado em sementes de pimenta-malagueta (Capsicum frutescens L.). Revista Ciência Agronômica, $36(1), 98-104$.

Venturin, A. Z., Lopes, J. C., Manhone, P. R., Zanotti, R. F. \& Carvalho, S. O. (2014). Superação de dormência de sementes de Capsicum baccatum var. pendulum (pimenta dedo de moça). Brazilian Journal of Agriculture, 89(2), 117-124. https://doi.org/10.37856/bja.v89i2.44

Vieira, R. D., Penariol, A. L., Perecin, D. \& Panobianco, M. (2002). Condutividade elétrica e teor de água inicial das sementes de soja. Pesq. agropec. bras. 37(9). https://doi.org/10.1590/S0100-204X2002000900018

Warraich, E. A., Basra, S. M. A., Ahmad, N. \& Aftab, M. (2002). Effect of nitrogen on grain qualty and vigour in wheat (tritticum aestivum L.). International Journal of Agricultura \& Biology, 4, 517-520. https://www.researchgate.net/publication/237752813_Effect_of_Nitrogen_on_Grain_Quality_and_Vi gour_in_Wheat_Triticum_aestivum_L.

Zucareli, C., Panoff, B., Portugal, G., \& Fonseca, I. C. B. (2012). Doses e épocas de aplicação de nitrogênio em cobertura na qualidade fisiológica de sementes de milho doce. Revista Brasileira de Sementes, 34(3), 480-487. https://doi.org/10.1590/S0101-31222012000300016 